\title{
The center of some braid groups and the Farrell cohomology of certain pure mapping class groups
}

\author{
YU QING CHEN \\ HENRY H GLOVER \\ CRAIG A JENSEN
}

\begin{abstract}
In this paper we first show that many braid groups of low genus surfaces have their centers as direct factors. We then give a description of centralizers and normalizers of prime order elements in pure mapping class groups of surfaces with spherical quotients using automorphism groups of fundamental groups of the quotient surfaces. As an application, we use these to show that the $p$-primary part of the Farrell cohomology groups of certain mapping class groups are elementary abelian groups. At the end we compute the $p$-primary part of the Farrell cohomology of a few pure mapping class groups.
\end{abstract}

20F36, 20J06

\section{Introduction}

Braid groups were first introduced by Artin $[1 ; 2 ; 3]$ for the purpose of studying knots and links. A geometric braid on $n$ strings can be defined as follow. Let $E=\mathbb{E}^{2} \times I$, where $\mathbb{E}^{2}$ is the Euclidean plane and $I$ is the unit interval $[0,1]$. Let $P_{i}=(i, 0,1) \in \mathbb{E}^{2} \times$ $\{1\}$ and $P_{i}^{\prime}=(i, 0,0) \in \mathbb{E}^{2} \times\{0\}$ for $i=1,2, \ldots, n$. An $n$-string $s=\left\{s_{1}, s_{2}, \ldots, s_{n}\right\}$ in $E$ is a set of continuous maps $s_{i}:[0,1] \rightarrow E, i=1,2, \ldots, n$, satisfying

(I) $s_{i}(0)=P_{i}$ and $s_{i}(1)=P_{\sigma(i)}^{\prime}, i=1,2, \ldots, n$, where $\sigma$ is a permutation of the set $\{1,2, \ldots, n\}$, and

(II) each plane $\mathbb{E}^{2} \times\{t\}$ in $E, 0 \leqslant t \leqslant 1$, intersects each string $s_{i}([0,1])$ in exactly one point for $i=1,2, \ldots, n$ and intersects strings $\bigcup_{i=1}^{n} s_{i}([0,1])$ in exactly $n$ distinct points.

Two $n$-strings $s$ and $s^{\prime}$ given by the same permutation $\sigma$ of $\{1,2, \ldots, n\}$ are said to be equivalent if there is a homotopy $H=\left(H_{1}, H_{2}, \ldots, H_{n}\right)$, where $H_{i}, i=1,2, \ldots, n$, are maps

$$
H_{i}:[0,1] \times[0,1] \rightarrow E,
$$


such that

$$
\left\{\begin{array}{l}
H_{i}(u, 0)=s_{i}(u), \\
H_{i}(u, 1)=s_{i}^{\prime}(u), \\
H_{i}(0, v)=P_{i}, \\
H_{i}(1, v)=P_{\sigma(i)}^{\prime},
\end{array}\right.
$$

and such that for every $v \in[0,1], H_{v}=\left\{H_{1}(\cdot, v), H_{2}(\cdot, v), \ldots, H_{n}(\cdot, v)\right\}$ is an $n$-string. Then a geometric braid on $n$ strings is an equivalence class of $n$-strings. Geometric braids can be presented pictorially by two horizontal lines with the points $P_{i}$ on the top line and $P_{i}^{\prime}$ on the bottom line and strings going from the $P_{i}$ to the $P_{i}^{\prime}$. The set of braids on $n$ strings is denoted by $B_{n}$. We can define a product of two braids in $B_{n}$ using these pictures. Let $b_{1}$ and $b_{2}$ be two geometric braids on $n$ strings. The picture of $b=b_{1} b_{2}$ is obtained as follows: placing the picture of $b_{1}$ above $b_{2}$ and identifying the bottom line of $b_{1}$ with the top line of $b_{2}$ in such a way that the points $P_{1}^{\prime}, P_{2}^{\prime} \ldots, P_{n}^{\prime}$ in $b_{1}$ match $P_{1}, P_{2}, \ldots, P_{n}$ in $b_{2}$. Then removing the common line between $b_{1}$ and $b_{2}$. It is easy to check that this product is well defined. Therefore $B_{n}$ is a group which is called Artin braid group on $n$ strings. A braid with the trivial permutation $\sigma$ is called a pure braid. The set of pure braids forms a normal subgroup of $B_{n}$ and is denoted by $P_{n}$. It is called the pure Artin braid group. There is an exact sequence

$$
1 \rightarrow P_{n} \rightarrow B_{n} \rightarrow \Sigma_{n} \rightarrow 1
$$

where $\Sigma_{n}$ is the symmetry group on $n$ symbols. Given a subgroup $G$ of $\Sigma_{n}$, we define the $G$-Artin braid group $B_{n}^{G}$ on $n$ strings to be the subgroup of $B_{n}$ such that for all braids in $B_{n}^{G}$, the permutations $\sigma$ in (I) are from $G$. Clearly, $P_{n} \leqslant B_{n}^{G} \leqslant B_{n}$ and $B_{n}^{\Sigma_{n}}=B_{n}$ and $B_{n}^{\{1\}^{n}}=P_{n}$, where $\{1\}^{k}$ is the trivial subgroup of $\Sigma_{k}$. The group $B_{n}^{G}$ is simply the pre-image of $G$ in $B_{n}$ of the exact sequence (1) and one has an exact sequence

$$
1 \rightarrow P_{n} \rightarrow B_{n}^{G} \rightarrow G \rightarrow 1
$$

Another family of groups that we are interested in are the mapping class groups. They are closely related to braid groups. Let $S_{g}^{n}$ denote the orientable surface of genus $g$ with $n$ punctures. The mapping class group $\Gamma_{g}^{n}$ of $S_{g}^{n}$ is defined to be the isotopy classes of orientation preserving homeomorphisms from $S_{g}^{n}$ to itself. It is the discrete group of connected components of the group of orientation preserving homeomorphisms of $S_{g}^{n}$. For other equivalent definitions of $\Gamma_{g}^{n}$ and the properties of $\Gamma_{g}^{n}$ with $n=0$, we refer the reader to the survey paper of Mislin [14]. Each $\Gamma_{g}^{n}$ contains a normal subgroup consisting of isotopy classes of orientation preserving homeomorphisms of $S_{g}^{n}$ which 
point-wise fix the punctures. This subgroup is called pure mapping class group of $S_{g}^{n}$ and is denoted by $P \Gamma_{g}^{n}$. Similar to the braid groups, we have the exact sequence

$$
1 \rightarrow P \Gamma_{g}^{n} \rightarrow \Gamma_{g}^{n} \rightarrow \Sigma_{n} \rightarrow 1
$$

For each subgroup $G$ of $\Sigma_{n}$, we can also define the $G$-mapping class group of $S_{g}^{n}$, $\Gamma_{g}^{n, G}$, to be the pre-image of $G$ in $\Gamma_{g}^{n}$ of the exact sequence (2). Then one has $P \Gamma_{g}^{n} \leqslant \Gamma_{g}^{n, G} \leqslant \Gamma_{g}^{n}$, and $\Gamma_{g}^{n, \Sigma_{n}}=\Gamma_{g}^{n}$ and $\Gamma_{g}^{n,\{1\}^{n}}=P \Gamma_{g}^{n}$, and the exact sequence

$$
1 \rightarrow P \Gamma_{g}^{n} \rightarrow \Gamma_{g}^{n, G} \rightarrow G \rightarrow 1
$$

In [11], Lu proved that $P \Gamma_{g}^{n}$ has periodic cohomology with period 2 for all $g \geqslant 1$ and $n \geqslant 1$, that is, $\hat{H}^{i+2}\left(P \Gamma_{g}^{n}, \mathbb{Z}\right) \cong \hat{H}^{i}\left(P \Gamma_{g}^{n}, \mathbb{Z}\right)$. She also computed the $p$-primary part of the Farrell cohomology of punctured pure mapping class group $\hat{H}^{*}\left(P \Gamma_{n(p-1) / 2}^{m}, \mathbb{Z}\right)_{(p)}$ for some small values of $n(n \leqslant 3)$ as well as $\hat{H}^{*}\left(P \Gamma_{g}^{n}, \mathbb{Z}\right)$ for small values of $g$ $(g \leqslant 3)$, see $\mathrm{Lu}[11 ; 12 ; 13]$.

In this paper, we first show in Theorem 3.3, Theorem 3.10 and Theorem 3.11 that for many braid groups of $S_{0}^{1}$ (2-sphere with 1 puncture), $S_{0}^{0}$ (2-sphere with no punctures) and $S_{1}^{0}$ (torus with no punctures) (see the definition of these groups in Section 2), their centers are actually direct factors of these groups. We then give in Theorem 5.6 an algebraic description of centralizers and normalizers of elements of order $p$ in $P \Gamma_{g}^{m}$ when the quotient surfaces are punctured 2 -spheres. Combining the splitting of braid groups and the alternative description of normalizers, we are able to obtain structural information about the $p$-primary part of the Farrell cohomology of $P \Gamma_{n(p-1) / 2}^{m}$ for certain values of $n$ and $m$ in Lemma 5.4. At the end of the of the paper in Theorem 5.8, we give an explicit computation of $\hat{H}^{i}\left(P \Gamma_{n(p-1) / 2}^{n+1}, \mathbb{Z}\right)_{(p)}, \widehat{H}^{i}\left(P \Gamma_{n(p-1) / 2}^{n+2}, \mathbb{Z}\right)_{(p)}$, $\widehat{H}^{i}\left(P \Gamma_{n}^{2 n+1}, \mathbb{Z}\right)_{(2)}$ and $\widehat{H}^{i}\left(P \Gamma_{n}^{2 n+2}, \mathbb{Z}\right)_{(2)}$.

The rest of the paper is organized as follow. In Section 2 we review some well known facts about braid groups and mapping class groups. In Section 3 we prove that the braid groups of $S_{0}^{1}, S_{0}^{0}$ and $S_{1}^{0}$ are the direct product of their centers with other braid groups or mapping class groups. In Section 4, we compute centralizers and normalizers of elements of prime order in pure mapping class groups, provided that the quotient surfaces are punctured 2-spheres. In Section 5, we study the $p$-primary part of the Farrell cohomology of pure mapping class groups and compute the $p$-primary part of Farrell cohomology mentioned above. 


\section{Braid groups and mapping class groups of surfaces}

This section contains the descriptions of braid groups and mapping class groups of surfaces which are different from the ones given in the introduction. The relations between pure braid groups and pure mapping class groups of the punctured 2-spheres are discussed at the end of this section.

The use of configuration spaces to describe braid groups seems to be suggested by Fox. This approach enables one to define braid groups of any topological space, and the braid groups defined in Section 1 are simply the braid groups of Euclidean plane.

Let $X$ be a topological space. Its $n$-fold configuration space $F_{n}(X)$ for $n \geq 0$ is defined by

$$
F_{n}(X)=\left\{\left(x_{1}, x_{2}, \ldots, x_{n}\right) \in \prod_{i=1}^{n} X \mid x_{i} \neq x_{j} \text { for all } i \neq j\right\}
$$

with the convention that when $n=0$, the space $F_{0}(X)$ consists of a single point and when $n=1$, the space $F_{1}(X)=X$ for any space $X$. Each subgroup $G$ of $\Sigma_{n}$ has a natural free action on $F_{n}(X)$ by permuting the coordinates. We denote by $F_{n}^{G}(X)$ the quotient space $F_{n}(X) / G$, that is,

$$
F_{n}^{G}(X)=\left\{\left(x_{1}, x_{2}, \ldots, x_{n}\right)^{G} \mid x_{i} \neq x_{j} \text { for all } i \neq j\right\},
$$

where $\left(x_{1}, x_{2}, \ldots, x_{n}\right)^{G}$ is the $G$-orbit of $\left(x_{1}, x_{2}, \ldots, x_{n}\right)$ in $F_{n}(X)$. Then the $G-$ braid group of $X$ on $n$ strings is defined to be the fundamental group $\pi_{1}\left(F_{n}^{G}(X)\right)$ of $F_{n}^{G}(X)$. When $G=\{1\}^{n}, F_{n}^{\{1\}^{n}}(X)=F_{n}(X)$ and $\pi_{1}\left(F_{n}(X)\right)$ is called the pure braid group of $X$ on $n$ strings. When $G=\Sigma_{n}, \pi_{1}\left(F_{n}^{\Sigma_{n}}(X)\right)$ is called full braid group of $X$ on $n$ strings. Obviously, the natural projection from $F_{n}(X)$ to $F_{n}^{G}(X)=F_{n}(X) / G$ is a regular covering with deck transformation group $G$. Thus we have an exact sequence of groups

$$
1 \rightarrow \pi_{1}\left(F_{n}(X)\right) \rightarrow \pi_{1}\left(F_{n}^{G}(X)\right) \rightarrow G \rightarrow 1 .
$$

If the space $X$ is the Euclidean plane, or equivalently, the 1-punctured 2-sphere $S_{0}^{1}$, then the braid group $\pi_{1}\left(F_{n}^{G}\left(S_{0}^{1}\right)\right)$ of $S_{0}^{1}$ is precisely the $G$-Artin braid group $B_{n}^{G}$. The subgroup $\pi_{1}\left(F_{n}\left(S_{0}^{1}\right)\right)$ is the pure Artin braid group $P_{n}$. For the connection between the two definitions of the Artin braid groups we refer the reader to Birman [4]. For a fixed set of distinguished points $Q_{m}=\left\{x_{1}, x_{2}, \ldots, x_{m}\right\}$ of a topological space $X$, we define

$$
F_{m, n}(X)=F_{n}\left(X-Q_{m}\right)
$$

and for any subgroup $G$ of $\Sigma_{n}$ we define

$$
F_{m, n}^{G}(X)=F_{n}^{G}\left(X-Q_{m}\right) .
$$

Algebraic $\mathcal{E}$ Geometric Topology, Volume 7 (2007) 
Note that the topological type of the configuration space $F_{m, n}(X)$ is independent of the choice of the particular points in the set $Q_{m}$, since one may always find an isotopy of $X$ which deforms one such set to another. Let $n=s+t, G=G_{s} \times G_{t}$ for some subgroups $G_{s}$ in $\Sigma_{s}$ and $G_{t}$ in $\Sigma_{t}$. The following theorem, which is a slight generalization of a version in Fadell-Neuwirth [10], describes a link among various configuration spaces $F_{m, n}^{G}(X)$ for a given space $X$.

Theorem 2.1 Fadell and Neuwirth [10] Let $\pi: F_{m, s+t}^{G_{s} \times G_{t}}(X) \rightarrow F_{m, s}^{G_{s}}(X)$ be the projection defined by

$$
\pi\left(\left(x_{1}, x_{2}, \ldots, x_{s+t}\right)^{G_{s} \times G_{t}}\right)=\left(x_{1}, x_{2}, \ldots, x_{s}\right)^{G_{s}} .
$$

Then $\pi$ is a locally trivial fibration with fibre $F_{m+s, t}^{G_{t}}(X)$.

Remark 2.2 The fibration is usually not globally trivial.

We will use this theorem to establish a connection between $\pi_{1}\left(F_{n}^{G}\left(S_{0}^{1}\right)\right)$ and $\pi_{1}\left(F_{n}^{G}\left(S_{0}^{0}\right)\right)$.

In [1], Artin gave an algebraic description of the Artin braid groups which is now called the Artin representation.

Theorem 2.3 Artin [1] Let $\operatorname{Aut}\left(F_{n}\right)$ denote the group of automorphisms of the free group with generators $x_{1}, x_{2}, \ldots x_{n}$. The Artin braid group

$$
\begin{aligned}
B_{n} \cong & \left\{\gamma \in \operatorname{Aut}\left(F_{n}\right) \mid \gamma\left(x_{i}\right) \text { is conjugate to } x_{\sigma(i)} \text { for some permutation } \sigma\right. \text { of } \\
& \left.\{1,2, \ldots, n\} \text { and for all } i=1,2, \ldots, n \text { and } \gamma\left(x_{1} x_{2} \cdots x_{n}\right)=x_{1} x_{2} \cdots x_{n}\right\} .
\end{aligned}
$$

It follows easily from Theorem 2.3 that for any subgroup $G$ of $\Sigma_{n}$, the $G$-Artin braid group

$$
\begin{aligned}
B_{n}^{G} \cong & \left\{\gamma \in \operatorname{Aut}\left(F_{n}\right) \mid \gamma\left(x_{i}\right) \text { is conjugate to } x_{\sigma(i)} \text { for some } \sigma \in G\right. \\
& \left.\quad \text { and for all } i=1,2, \ldots, n \text { and } \gamma\left(x_{1} x_{2} \cdots x_{n}\right)=x_{1} x_{2} \cdots x_{n}\right\} .
\end{aligned}
$$

The centers of $G$-Artin braid groups are infinite cyclic groups. Geometrically, these groups are generated by the braids with a full twist. If we use the Artin representation to describe these centers, they are precisely the intersection of the braid groups with the inner automorphism groups of those free groups.

Mapping class groups $\Gamma_{g}^{n, G}$ introduced in the previous section can also be given an algebraic description using automorphisms of fundamental groups of the surfaces $S_{g}^{n}$ 
as in Dicks-Formanek [9] and Zieschang [16]. The fundamental group of $S_{g}^{n}$ admits a presentation

$$
\pi_{1}\left(S_{g}^{n}\right)=\left\langle x_{1}, x_{2}, \ldots, x_{n}, a_{1}, b_{1}, a_{2}, b_{2}, \ldots, a_{g}, b_{g} \mid x_{1} x_{2} \cdots x_{n} \prod_{i=1}^{n}\left[a_{i}, b_{i}\right]=1\right\rangle,
$$

where $x_{1}, x_{2}, \ldots, x_{n}$ are the loops representing those punctures which are oriented in a consistent manner. For a subgroup $G$ of $\Sigma_{n}$, we define

$$
\begin{aligned}
\operatorname{Aut}_{+}^{G}\left(\pi_{1}\left(S_{g}^{n}\right)\right)=\left\{\gamma \in \operatorname{Aut}\left(\pi_{1}\left(S_{g}^{n}\right)\right) \mid \gamma\left(x_{i}\right) \text { is conjugate to } x_{\sigma(i)}\right. \\
\text { for some } \sigma \in G \text { and for all } i=1,2, \ldots, n\} .
\end{aligned}
$$

Then the $G$-mapping class group of $S_{g}^{n}$

$$
\Gamma_{g}^{n, G} \cong \operatorname{Aut}_{+}^{G}\left(\pi_{1}\left(S_{g}^{n}\right)\right) / \operatorname{Inn}\left(\pi_{1}\left(S_{g}^{n}\right)\right),
$$

where $\operatorname{Inn}\left(\pi_{1}\left(S_{g}^{n}\right)\right)$ is the inner automorphism group of $\pi_{1}\left(S_{g}^{n}\right)$.

If we set $g=0$, by comparing the algebraic descriptions of $B_{n}^{G}$ and $\Gamma_{0}^{n, G}$, it is obvious that $B_{n}^{G} / Z\left(B_{n}^{G}\right) \cong \Gamma_{0}^{n+1, G \times\{1\}}$, where $Z\left(B_{n}^{G}\right)$ is the center of $B_{n}^{G}$.

We end this section with the following proposition that summarizes the known relation between the braid groups of $S_{0}^{1}$ and $S_{0}^{0}$ and the mapping class groups of the punctured 2-sphere. Some version of this can be found in Biran [4] and Fadell-Neuwirth [10].

\section{Proposition 2.4}

(1) The center $Z\left(B_{n}^{G}\right)$ of $B_{n}^{G}$ is an infinite cyclic group and $B_{n}^{G} / Z\left(B_{n}^{G}\right) \cong$ $\Gamma_{0}^{n+1, G \times\{1\}} ;$

(2) the center $Z\left(\pi_{1}\left(F_{n}^{G}\left(S_{0}\right)\right)\right)$ of $\pi_{1}\left(F_{n}^{G}\left(S_{0}\right)\right)$ is a cyclic group of order 2 and $\pi_{1}\left(F_{n}^{G}\left(S_{0}\right)\right) / Z\left(\pi_{1}\left(F_{n}^{G}\left(S_{0}\right)\right)\right) \cong \Gamma_{0}^{n, G}$.

\section{The center of braid groups}

In this section we will show that under some mild conditions, the center of many braid groups of $S_{0}^{1}, S_{0}^{0}, S_{1}^{0}$ are direct factors of these groups. Let us recall a few more well known facts about the braid groups. The configuration space $F_{n}\left(S_{0}^{1}\right)$, which we used to define the pure Artin group $P_{n}$, is an Eilenberg-MacLane space $K\left(P_{n}, 1\right)$ of $P_{n}$ since $\pi_{i}\left(F_{n}\left(S_{0}^{1}\right)\right)=0$ for $i \geqslant 2$ (see [4]). Also since $F_{n}\left(S_{0}^{1}\right)$ is a finite cover of $F_{n}^{G}\left(S_{0}^{1}\right)$ for any subgroup $G$ of $\Sigma_{n}$, one has $\pi_{i}\left(F_{n}^{G}\left(S_{0}^{1}\right)\right)=0$ for $i \geqslant 2$. This implies that $B_{n}^{G}$ is torsion free because it has finite cohomological dimension. In fact, if $S$ is a 
surface with $\pi_{2}(S)=1$, that is, $S \neq S_{0}^{0}$, the space $F_{n}^{G}(S)$ is an Eilenberg-MacLane space of $\pi_{1}\left(F_{n}^{G}(S)\right)$ for any $n$ and any subgroup $G$ of $\Sigma_{n}$. Hence these braid groups are of finite cohomological dimension and are torsion free. Also from the geometric definition of $P_{n}$, there is an exact sequence

$$
1 \rightarrow F_{n-1} \rightarrow P_{n} \rightarrow P_{n-1} \rightarrow 1,
$$

where the projection $P_{n} \rightarrow P_{n-1}$ is given by removing a string from $P_{n}$ and the kernel of this projection is isomorphic to the free group $F_{n-1}$ on $n-1$ generators. The above exact sequence splits. Therefore

$$
P_{n} \cong F_{n-1} \rtimes P_{n-1},
$$

where the action of $P_{n-1}$ on $F_{n-1}$ is given by the Artin representation. For example, $P_{3} \cong F_{2} \rtimes \mathbb{Z}$ and the action of $\mathbb{Z}$ on $F_{2}$ is given by an inner automorphism of $F_{2}$.

We now prove a simple lemma concerning the configuration spaces of topological groups.

Lemma 3.1 If $K$ is a topological group and $G$ is a subgroup of $\Sigma_{n}$ such that $G=$ $H \times\{1\}$, where $H$ is a subgroup of $\Sigma_{n-1}$, then

$$
F_{n}^{G}(K) \cong F_{n-1}^{H}(K-\{\mathbf{1}\}) \times K,
$$

where $\mathbf{1}$ is the identity element of $K$.

Proof We define

$$
\begin{aligned}
& u: F_{n}^{G}(K) \rightarrow F_{n-1}^{H}(K-\{\mathbf{1}\}) \times K, \\
& u\left(\left(k_{1}, k_{2}, \ldots, k_{n-1}, k_{n}\right)^{G}\right)=\left(\left(k_{1} k_{n}^{-1}, k_{2} k_{n}^{-1}, \ldots, k_{n-1} k_{n}^{-1}\right)^{H}, k_{n}\right)
\end{aligned}
$$

and

$$
\begin{aligned}
& v: F_{n-1}^{H}(K-\{\mathbf{1}\}) \times K \rightarrow F_{n}^{G}(K), \\
& v\left(\left(k_{1}, k_{2}, \ldots, k_{n-1}\right)^{H}, k_{n}\right)=\left(k_{1} k_{n}, k_{2} k_{n}, \ldots, k_{n-1} k_{n}, k_{n}\right)^{G} .
\end{aligned}
$$

It is easy to verify that $u$ and $v$ are continuous and inverse to each other.

Remark 3.2 Comparing this Lemma with Theorem 2.1, we can see that the fibration in Theorem 2.1 with $m=0, s=1$ and $t=n-1$ is usually not a trivial fibration, while Lemma 3.1 provides a trivial one when the space has a group structure.

Algebraic 83 Geometric Topology, Volume 7 (2007) 
Theorem 3.3 Let $G$ be a subgroup of $\Sigma_{n}$ such that $G=H \times\{1\}^{2}$, where $H$ is a subgroup of $\Sigma_{n-2}$. The $G$-Artin braid group

$$
B_{n}^{G} \cong \pi_{1}\left(F_{n-2}^{H}\left(S_{0}^{3}\right)\right) \times \mathbb{Z} \cong \Gamma_{0}^{n+1, G \times\{1\}} \times \mathbb{Z}
$$

for $n \geqslant 2$, where $G \times\{1\}$ is a subgroup of $\Sigma_{n+1}$.

Proof We use the complex number plane $\mathbb{C}$ for the Euclidean plane $\mathbb{E}^{2}$ and denote $\mathbb{C}-\{0\}$ by $\mathbb{C}^{*}$, the non-zero complex numbers. Then we have

$$
\begin{aligned}
B_{n}^{G} & =\pi_{1}\left(F_{n}^{G}(\mathbb{C})\right) \\
& \cong \pi_{1}\left(F_{n-1}^{H \times\{1\}}\left(\mathbb{C}^{*}\right) \times \mathbb{C}\right) \\
& =\pi_{1}\left(F_{n-1}^{H \times\{1\}}\left(\mathbb{C}^{*}\right)\right) \\
& \cong \pi_{1}\left(F_{n-2}^{H}\left(\mathbb{C}^{*}-\{1\}\right) \times \mathbb{C}^{*}\right) \\
& \cong \pi_{1}\left(F_{n-2}^{H}\left(\mathbb{C}^{*}-\{1\}\right)\right) \times \pi_{1}\left(\mathbb{C}^{*}\right) \\
& =\pi_{1}\left(F_{n-2}^{H}\left(S_{0}^{3}\right)\right) \times \mathbb{Z} .
\end{aligned}
$$

Here we used Lemma 3.1 twice, once for the additive group of $\mathbb{C}$ and once for the multiplicative group $\mathbb{C}^{*}$. The factor $\pi_{1}\left(\mathbb{C}^{*}\right) \cong \mathbb{Z}$ is obviously contained in the center $Z\left(B_{n}^{G}\right)$ of $B_{n}^{G}$. In order to show that it is actually the full center, we only need that $Z\left(B_{n}^{G}\right) \cong \mathbb{Z}$. By statement (1) of Proposition 2.4, we have

$$
B_{n}^{G} \cong \Gamma_{0}^{n+1, G \times\{1\}} \times \mathbb{Z} .
$$

Corollary 3.4 $P_{n} \cong P \Gamma_{0}^{n+1} \times \mathbb{Z}$.

Remark 3.5 We can also use the fact that $P_{n} \cong F_{n-1} \rtimes P_{n-1}$ to prove Corollary 3.4 by induction.

Remark 3.6 Note that in Theorem 3.3, we have $G=H \times\{1\}^{2}$, a subgroup of $\Sigma_{n}$ that fixes at least two elements. If $G$ fixes at most one element, that is, $G$ does not fix any elements or $G=H \times\{1\}$ with $H$ a subgroup of $\Sigma_{n-1}$ which does not fix any elements, then the braid group $B_{n}^{G}$ does not split.

Corollary 3.7 The space $F_{n-3}^{H}\left(S_{0}^{3}\right)$ is an Eilenberg-MacLane space of $\Gamma_{0}^{n, H \times\{1\}^{3}}$ for any subgroup $H$ of $\Sigma_{n-3}$. Therefore $\Gamma_{0}^{n, H \times\{1\}^{3}}$ has finite cohomological dimension and is torsion free. 
Remark 3.8 The corollary recovers the well known fact that $P \Gamma_{0}^{4} \cong F_{2}$, the free group on two generators. Furthermore, combining this fact with Corollary 3.4, we obtain that $P_{3} \cong F_{2} \times \mathbb{Z}$. This description of $P_{3}$ is simpler than the one given before Lemma 3.1.

Remark 3.9 When $H$ is a subgroup of $\Sigma_{n-2}$, the group $\Gamma_{0}^{n, H \times\{1\}^{2}}$ may contain torsion, and hence is not of finite cohomological dimension.

We now use Lemma 3.1 to give a similar decomposition of the $G$-braid group of the 2-sphere.

Theorem 3.10 Let $G$ be a subgroup of $\Sigma_{n}$ such that $G=H \times\{1\}^{3}$ for some subgroup $H$ of $\Sigma_{n-3}$. The $G$-braid group of the 2 -sphere

$$
\pi_{1}\left(F_{n}^{G}\left(S_{0}^{0}\right)\right) \cong \Gamma_{0}^{n, G} \times \mathbb{Z} / 2 \mathbb{Z}
$$

for $n \geqslant 3$.

Proof If we apply Theorem 2.1 with $m=0, s=1, t=n-1$ and $X=S_{0}^{0}$, we obtain a fibration

$$
\pi: F_{n}^{G}\left(S_{0}^{0}\right) \rightarrow S_{0}^{0}
$$

with fibre $F_{1, n-1}^{H \times\{1\}^{2}}\left(S_{0}^{0}\right)=F_{n-1}^{H \times\{1\}^{2}}\left(S_{0}^{1}\right)$. Thus we have an exact sequence of the homotopy groups of the fibration

$$
\begin{aligned}
1=\pi_{2}\left(F_{n-1}^{H \times\{1\}^{2}}\left(S_{0}^{1}\right)\right) \longrightarrow & \pi_{2}\left(F_{n}^{G}\left(S_{0}^{0}\right)\right) \longrightarrow \pi_{2}\left(S_{0}^{0}\right) \longrightarrow \\
& \pi_{1}\left(F_{n-1}^{H \times\{1\}^{2}}\left(S_{0}^{1}\right)\right) \longrightarrow \pi_{1}\left(F_{n}^{G}\left(S_{0}^{0}\right)\right) \longrightarrow \pi_{1}\left(S_{0}^{0}\right)=1 .
\end{aligned}
$$

Since $\pi_{2}\left(S_{0}^{0}\right) \cong \mathbb{Z}$ and, by Theorem 3.3, $\pi_{1}\left(F_{n-1}^{H \times\{1\}^{2}}\left(S_{0}^{1}\right)\right) \cong \Gamma_{0}^{n, G} \times \mathbb{Z}$, the above exact sequence is equivalent to

$$
1 \longrightarrow \pi_{2}\left(F_{n}^{G}\left(S_{0}^{0}\right)\right) \stackrel{i}{\longrightarrow} \mathbb{Z} \stackrel{j}{\longrightarrow} \Gamma_{0}^{n, G} \times \mathbb{Z} \stackrel{k}{\longrightarrow} \pi_{1}\left(F_{n}^{G}\left(S_{0}^{0}\right)\right) \longrightarrow 1 .
$$

The surjection $k$ maps the center of $\Gamma_{0}^{n, G} \times \mathbb{Z}$ onto the center of $\pi_{1}\left(F_{n}\left(S_{0}^{0}\right)\right)$ which is isomorphic to $\mathbb{Z} / 2 \mathbb{Z}$. Therefore we must have that $\pi_{2}\left(F_{n}^{G}\left(S_{0}^{0}\right)\right)=1$ and $j(\mathbb{Z})$ is a subgroup of index 2 of the center of $\Gamma_{0}^{n, G} \times \mathbb{Z}$. From this one can easily see that

$$
\pi_{1}\left(F_{n}^{G}\left(S_{0}^{0}\right)\right) \cong \Gamma_{0}^{n, G} \times \mathbb{Z} / 2 \mathbb{Z} .
$$

The last application of Lemma 3.1 is to prove the splitting of some braid groups of torus. 
Theorem 3.11 Let $G$ be a subgroup of $\Sigma_{n}$ such that $G=H \times\{1\}$ for some subgroup $H$ of $\Sigma_{n-1}$. The $G$-braid group of the torus

$$
\pi_{1}\left(F_{n}^{G}\left(S_{1}^{0}\right)\right) \cong \pi_{1}\left(F_{n-1}^{H}\left(S_{1}^{1}\right)\right) \times \mathbb{Z}^{2}
$$

for $n \geqslant 1$.

Proof Let $C=\left\{x \in \mathbb{C}^{*}|| x \mid=1\right\}$ be the unit circle in the complex number plane. Torus $S_{1}^{0}$ is homeomorphic to $C \times C$, which is a topological group. By Lemma 3.1,

$$
\begin{aligned}
\pi_{1}\left(F_{n}^{G}\left(S_{1}^{0}\right)\right) & =\pi_{1}\left(F_{n}^{G}(C \times C)\right) \\
& \cong \pi_{1}\left(F_{n-1}^{H}((C \times C)-\{(1,1)\}) \times(C \times C)\right) \\
& \cong \pi_{1}\left(F_{n-1}^{H}\left(S_{1}^{1}\right)\right) \times \pi_{1}(C \times C) \\
& \cong \pi_{1}\left(F_{n-1}^{H}\left(S_{1}^{1}\right)\right) \times \mathbb{Z}^{2}
\end{aligned}
$$

for $n \geqslant 1$.

\section{The centralizer and normalizer of elements of prime order in mapping class groups}

In this section we discuss the centralizer and normalizer of elements of prime order in mapping class groups $\Gamma_{g}^{n}$. Detailed discussion of conjugacy classes of prime order finite subgroup of $P \Gamma_{g}^{n}$ can be found in Lu $[11 ; 12]$. Let us first recall two theorems from Birman-Hilden [5].

Theorem 4.1 [5, Theorem 1] Let $\left(p, S_{g}^{m}, S_{h}^{n}\right)$ be a regular covering space, either branched or unbranched, with a finite group of covering transformations and a finite number of branch points. Let the covering transformations leave each branch point fixed. In the case of a branched covering, assume that $S_{g}^{m}$ is not homeomorphic to the closed sphere or closed torus. Let $g: S_{g}^{m} \rightarrow S_{g}^{m}$ be a fiber-preserving homeomorphism of $S_{g}^{m}$ which is isotopic to the identity map. Then $g$ is fiber-isotopic to the identity map.

The term "fiber-isotopic" means the intermediate homeomorphisms between $g$ and the identity map in the isotopy are all fiber-preserving.

Theorem 4.2 [5, Theorem 3] Let $t, h \in \Gamma_{g}^{0}$ such that $t^{k}=1$ and $h t h^{-1} \in\langle t\rangle$. There are homeomorphisms $\tilde{t}$ and $\tilde{h}$ form $S_{g}^{0}$ to itself representing $t$ and $h$ respectively such that $\tilde{t}^{k}=1$ and $\tilde{h} \tilde{t}^{-1} \in\langle\widetilde{t}\rangle$. 
The two theorems above imply that if $\alpha \in \Gamma_{g}^{0}$ is of finite order and it point-wise fixes all, say $n$, of its branch points, then the normalizer of $\alpha$ in $\Gamma_{g}^{0}$ is isomorphic to that of $\alpha$ in $\Gamma_{g}^{n}$ with branch points being the punctures. The isomorphism between the two normalizers is established by selecting homeomorphisms of surfaces using Theorem 4.2 and map them to each other and Theorem 4.1 ensures that the maps are well defined at the mapping class level. More generally, if we remove, or mark, $m$ branch points beforehand, the same argument implies the following.

Proposition 4.3 Let $m \geqslant 0, g \geqslant 0$ be integers such that $(m, g) \neq(0,0),(0,1)$. Let $\alpha \in P \Gamma_{g}^{m}$ be of finite order such that there are exactly $n$ branch points in $S_{g}^{m}$ which are point-wise fixed by $\alpha$. By removing these $n$ additional points from $S_{g}^{m}$, one has $\alpha \in \Gamma_{g}^{n+m, \Sigma_{n} \times\{1\}^{m}}$ and the normalizer of $\alpha$ in $P \Gamma_{g}^{m}$ is isomorphic to that of $\alpha$ in $\Gamma_{g}^{n+m, \Sigma_{n} \times\{1\}^{m}}$.

Proposition 4.3 transfers the problem of finding the normalizer of a cyclic branched covering map to that of a cyclic regular covering map. Using this regular covering map, we can obtain a more detailed description of these normalizers when the quotient surface is homeomorphic to a punctured 2-sphere. This was partially done in Birman-Hilden [5] and Xia [15] by showing that there is an imbedding of the reduced normalizer of $\langle\alpha\rangle$, that is, the normalizer of $\langle\alpha\rangle$ modulo $\langle\alpha\rangle$, into the mapping class group of the quotient surface. We will describe the normalizer of $\langle\alpha\rangle$ in terms of the automorphism group of the fundamental group of the quotient surface.

Assume that $\langle\alpha\rangle \cong \mathbb{Z} / p \mathbb{Z}$ is a subgroup of $\Gamma_{g}^{n+m, \Sigma_{n} \times\{1\}^{m}}$ with no additional fixed points and $S_{g}^{n+m} /\langle\alpha\rangle$ is homeomorphic to $S_{h}^{n+m}$, where the numbers $g, h, n+$ $m$ and $p$ must satisfy the Riemann-Hurwitz formula $2 g-2=p(2 h-2)+(n+$ $m)(p-1)$. Let $f: \pi_{1}\left(S_{h}^{n+m}\right) \rightarrow\langle\alpha\rangle$ be the group homomorphism obtained from the regular covering $S_{g}^{n+m} \rightarrow S_{h}^{n+m}$ and $x_{1}, x_{2}, \ldots, x_{n+m}$ be the elements in $\pi_{1}\left(S_{h}^{m+n}\right)$ that represents the $n+m$ punctures. Then the values $f\left(x_{i}\right), i=1,2, \ldots, n+m$, satisfy $f\left(x_{1}\right)+f\left(x_{1}\right)+\cdots+f\left(x_{n+m}\right)=0$ and $f\left(x_{i}\right) \neq 0$ for all $1 \leqslant i \leqslant n+$ $m$. We denote by $(\mathbb{Z} / p \mathbb{Z})^{*}$ the non-zero elements in $\mathbb{Z} / p \mathbb{Z}$ and call the vector $F_{\alpha}=\left(f\left(x_{1}\right), f\left(x_{2}\right), \ldots, f\left(x_{n+m}\right)\right) \in\left((\mathbb{Z} / p \mathbb{Z})^{*}\right)^{n+m}$ the fixed point data vector of $\alpha$. Given a permutation $\sigma \in \Sigma_{n+m}$ and an element $k \in(\mathbb{Z} / p \mathbb{Z})^{*}$ define

$$
\begin{aligned}
\sigma\left(F_{\alpha}\right) & =\left(f\left(x_{\sigma(1)}\right), f\left(x_{\sigma(2)}\right), \ldots, f\left(x_{\sigma(n+m)}\right)\right) \in\left((\mathbb{Z} / p \mathbb{Z})^{*}\right)^{n+m} \text { and } \\
k F_{\alpha} & =\left(k f\left(x_{1}\right), k f\left(x_{2}\right), \ldots, k f\left(x_{n+m}\right)\right) \in\left((\mathbb{Z} / p \mathbb{Z})^{*}\right)^{n+m} .
\end{aligned}
$$

Let

$$
\begin{aligned}
& \Sigma_{\alpha}^{N}=\left\{\gamma \in \Sigma_{n} \times\{1\}^{m} \mid \gamma\left(F_{\alpha}\right)=k F_{\alpha} \text { for some } k \in(\mathbb{Z} / p \mathbb{Z})^{*}\right\}, \\
& \Sigma_{\alpha}^{C}=\left\{\gamma \in \Sigma_{n} \times\{1\}^{m} \mid \gamma\left(F_{\alpha}\right)=F_{\alpha}\right\} .
\end{aligned}
$$


We call $\Sigma_{\alpha}^{N}$ the normalizing permutation group of $\alpha$ and $\Sigma_{\alpha}^{C}$ the centralizing permutation group of $\alpha$. For each $t \in(\mathbb{Z} / p \mathbb{Z})^{*}$, let $l_{t}=\left|\left\{1 \leqslant i \leqslant n \mid f\left(x_{i}\right)=t\right\}\right|$. We observe from the definition that $\Sigma_{\alpha}^{C}=\Sigma_{l_{1}} \times \Sigma_{l_{2}} \times \cdots \times \Sigma_{l_{p-1}} \times\{1\}^{m}$. Consider the following commutative diagram

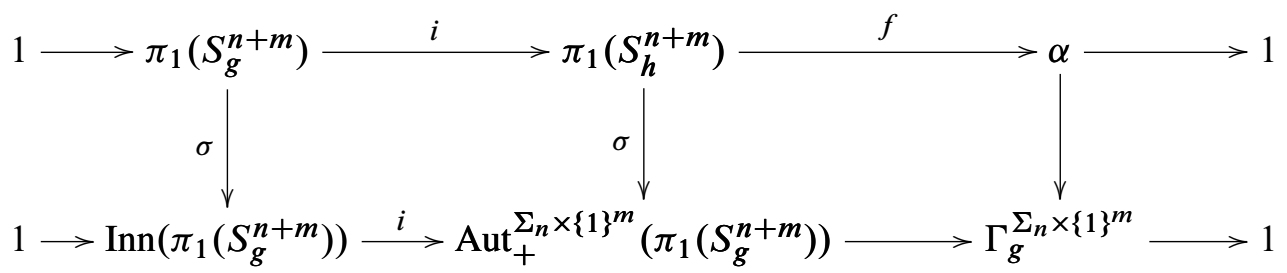

where the imbedding $\sigma$ (as long as $S_{g}^{n+m}$ and $S_{h}^{n+m}$ are not $S_{0}^{1}, S_{0}^{0}$ or $S_{1}^{0}$ ) is given by the conjugation action on $\pi_{1}\left(S_{g}^{m+n}\right)$, that is, for every $u \in \pi_{1}\left(S_{h}^{n+m}\right)$, $\sigma(u)(y)=i^{-1}\left(u i(y) u^{-1}\right)$ for all $y \in \pi_{1}\left(S_{g}^{n+m}\right)$. We know that the normalizer of $\langle\alpha\rangle$ in $\Gamma_{g}^{\Sigma_{n} \times\{1\}^{m}}$ is isomorphic to that of $\sigma\left(\pi_{1}\left(S_{h}^{n+m}\right)\right)$ in $\operatorname{Aut}_{+}^{\Sigma_{n} \times\{1\}^{m}}\left(\pi_{1}\left(S_{g}^{n+m}\right)\right)$ modulo $i\left(\operatorname{Inn}\left(\pi_{1}\left(S_{g}^{n+m}\right)\right)\right)$.

Lemma 4.4 The normalizer of $\sigma\left(\pi_{1}\left(S_{h}^{n+m}\right)\right)$ in $\mathrm{Aut}_{+}^{\Sigma_{n} \times\{1\}^{m}}\left(\pi_{1}\left(S_{g}^{n+m}\right)\right)$ is isomorphic to Aut $_{+}^{\Sigma_{\alpha}^{N}}\left(\pi_{1}\left(S_{h}^{n+m}\right)\right)$ when $h=0$.

Proof Let

$$
K=\left\{\gamma \in \operatorname{Aut}_{+}^{\Sigma_{n} \times\{1\}^{m}}\left(\pi_{1}\left(S_{h}^{n+m}\right)\right) \mid \gamma\left(i\left(\pi_{1}\left(S_{g}^{n+m}\right)\right)\right)=i\left(\pi_{1}\left(S_{g}^{n+m}\right)\right)\right\}
$$

and $J$ be the normalizer of $\sigma\left(\pi_{1}\left(S_{h}^{n+m}\right)\right)$ in $\operatorname{Aut}_{+}^{\Sigma_{n} \times\{1\}^{m}}\left(\pi_{1}\left(S_{g}^{n+m}\right)\right)$. We define two homomorphisms $\Phi: J \rightarrow K$ and $\Psi: K \rightarrow J:$

$$
\begin{aligned}
& \text { for every } x \in J, \Phi(x)(u)=\sigma^{-1}\left(x \sigma(u) x^{-1}\right) \text { for all } u \in \pi_{1}\left(S_{h}^{n+m}\right) \text {; } \\
& \text { for every } y \in K, \Psi(y)(v)=i^{-1}(y(i(v))) \text { for all } v \in \pi_{1}\left(S_{g}^{n+m}\right) .
\end{aligned}
$$

It can be routinely checked that $\Phi$ and $\Psi$ are inverse to each other. Now we need to show that $K=\operatorname{Aut}_{+}^{\Sigma_{\alpha}^{N}}\left(\pi_{1}\left(S_{h}^{n+m}\right)\right)$ when $h=0$. Recall that

$$
\pi_{1}\left(S_{0}^{n+m}\right)=\left\langle x_{1}, x_{2}, \ldots, x_{n+m} \mid x_{1} x_{2} \cdots x_{n+m}=1\right\rangle .
$$

Let $H$ be the normal closure of $\left\{t^{p} \mid t \in \pi_{1}\left(S_{0}^{n+m}\right)\right\}$ in $\pi_{1}\left(S_{0}^{n+m}\right)$ and $M=$ $H\left[\pi_{1}, \pi_{1}\right]$, where $\left[\pi_{1}, \pi_{1}\right]$ is the commutator subgroup of $\pi_{1}\left(S_{0}^{n+m}\right)$. Then $M$ is a characteristic subgroup of $\pi_{1}\left(S_{0}^{n+m}\right)$ and the homomorphism $f$ in the commutative 
diagram factors through

$$
\pi_{1}\left(S_{0}^{n+m}\right) / M=\bigoplus_{i=1}^{n+m} \mathbb{Z} / p \mathbb{Z} x_{i} /\left\langle x_{1}+x_{2}+\cdots+x_{n+m}\right\rangle
$$

An automorphism in $\mathrm{Aut}_{+}^{\Sigma_{n} \times\{1\}^{m}}\left(\pi_{1}\left(S_{0}^{n+m}\right)\right)$ fixes the subgroup $i\left(\pi_{1}\left(S_{g}^{n+m}\right)\right)=$ $\operatorname{Ker}(f)$ if and only if its induced automorphism in $\operatorname{Aut}\left(\pi_{1}\left(S_{0}^{n+m}\right) / M\right)$ fixes the kernel of

$$
\pi_{1}\left(S_{0}^{n+m}\right) / M \rightarrow\langle\alpha\rangle,
$$

which is given by

$$
\begin{aligned}
\left\{t_{1} x_{1}+t_{2} x_{2}+\cdots+t_{n+m} x_{n+m} \in\right. & \bigoplus_{i=1}^{n+m} \mathbb{Z} / p \mathbb{Z} x_{i} /\left\langle x_{1}+x_{2}+\cdots+x_{n+m}\right\rangle \\
& \left.\mid t_{1} f\left(x_{1}\right)+t_{2} f\left(x_{2}\right)+\cdots+t_{n+m} f\left(x_{n+m}\right)=0\right\} .
\end{aligned}
$$

In other words the kernel is the hyperplane $F_{\alpha}^{\perp}$ in $\pi_{1}\left(S_{0}^{n+m}\right) / M$ that is orthogonal to the fixed point data vector $F_{\alpha}$ of $\alpha$. On the other hand, every automorphism in the group $\operatorname{Aut}_{+}^{\Sigma_{n} \times\{1\}^{m}}\left(\pi_{1}\left(S_{0}^{n+m}\right)\right)$ induces a permutation automorphism of $\pi_{1}\left(S_{0}^{n+m}\right) / M$ which permutes the elements $x_{1}, x_{2}, \ldots, x_{n}$, and every such permutation automorphism in $\Sigma_{n} \times\{1\}^{m}$ is indeed induced by some automorphism in $\operatorname{Aut}_{+}^{\Sigma_{n} \times\{1\}^{m}}\left(\pi_{1}\left(S_{0}^{n+m}\right)\right)$. If $\gamma \in \Sigma_{n} \times\{1\}^{m}$, one has $\gamma\left(F_{\alpha}^{\perp}\right)=\gamma\left(F_{\alpha}\right)^{\perp}$. Hence $\gamma\left(F_{\alpha}^{\perp}\right)=F_{\alpha}^{\perp}$ if and only if $\gamma\left(F_{\alpha}\right)^{\perp}=F_{\alpha}^{\perp}$, or equivalently $\gamma \in \Sigma_{\alpha}^{N}$ and $K=$ $\operatorname{Aut}_{+}^{\Sigma_{\alpha}^{N}}\left(\pi_{1}\left(S_{0}^{n+m}\right)\right)$, when $h=0$.

Remark 4.5 The group $K$ in the proof is the group that maps loops that lift to loops to loops that lift to loops as all such loops form the group $i\left(\pi_{1}\left(S_{g}^{n+m}\right)\right)$. This property was used in $[5 ; 15]$.

Combining Proposition 4.3 and Lemma 4.4, we have proved the following theorem.

Theorem 4.6 Let $m \geqslant 0, g \geqslant 0$ be integers such that $(m, g) \neq(0,0),(0,1)$. Let $\alpha \in P \Gamma_{g}^{m}$ be an element of prime order $p$ with exactly $n$ branch points in $S_{g}^{m}$. Let $\Sigma_{\alpha}^{N}$ and $\Sigma_{\alpha}^{C}$ be the normalizing and centralizing permutation groups of $\alpha$ respectively. If the quotient surface $S_{g}^{m} /\langle\alpha\rangle$ is homeomorphic to $S_{0}^{m}$, then the normalizer of $\alpha$ in $P \Gamma_{g}^{m}$ is isomorphic to $\operatorname{Aut}_{+}^{\Sigma_{\alpha}^{N}}\left(\pi_{1}\left(S_{0}^{n+m}\right)\right) / \operatorname{Inn}\left(\pi_{1}\left(S_{g}^{n+m}\right)\right)$ and the centralizer of $\alpha$ in $P \Gamma_{g}^{m}$ is isomorphic to $\operatorname{Aut}_{+}^{\Sigma_{\alpha}^{C}}\left(\pi_{1}\left(S_{0}^{n+m}\right)\right) / \operatorname{Inn}\left(\pi_{1}\left(S_{g}^{n+m}\right)\right)$. 


\section{The $p$-primary part of the Farrell cohomology of certain pure mapping class groups}

The mapping class groups are known to have finite virtual cohomological dimensions (vcd) (see Lu [12], Mislin [14]), that is, they have subgroups of finite index which have finite cohomological dimensions. For group $\Gamma$ of finite vcd, the ordinary cohomology and Farrell cohomology are isomorphic above the vcd, that is,

$$
H^{k}(\Gamma, M) \cong \hat{H}^{i}(\Gamma, M)
$$

for all $\Gamma$-module $M$ and all $i>\operatorname{vcd}(\Gamma)$. Such a group $\Gamma$ is said to have $p$-periodic cohomology for some prime $p$ if there is a positive integer $d$ such that $\hat{H}^{i}(\Gamma, M)_{(p)} \cong$ $\hat{H}^{i+d}(\Gamma, M)_{(p)}$ for all integer $i$ and all $\Gamma$-module $M$, where $\hat{H}^{i}(\Gamma, M)_{(p)}$ is the $p$-primary part of $\hat{H}^{i}(\Gamma, M)$. By Brown [6, Theorem 6.7], a group of finite ved is $p$-periodic for a prime $p$ if and only if $\Gamma$ is of $p$-rank 1 , that is, every finite elementary abelian $p$-subgroup is rank 1 . The following theorem is needed in our study of the cohomology groups of certain pure mapping class groups.

Theorem 5.1 [6, Corollary 7.4] Suppose that $\Gamma$ is of finite vcd and every elementary abelian $p$-subgroup of $\Gamma$ has rank $\leqslant 1$. Then

$$
\hat{H}^{i}(\Gamma, \mathbb{Z})_{(p)} \cong \prod_{P \in \wp} \hat{H}^{i}\left(N_{\Gamma}(P), \mathbb{Z}\right)_{(p)},
$$

where $\wp$ is a set of representatives for the conjugacy classes of subgroups of $\Gamma$ of order $p, N_{\Gamma}(P)$ is the normalizer of $P$ in $\Gamma$.

In this section we study the $p$-primary part of the Farrell cohomology of the pure mapping class groups. Lu $[11 ; 12 ; 13]$ determined almost all of the $p$-primary part of Farrell cohomology of pure mapping class groups of low genus, as well as $P \Gamma_{n(p-1) / 2}^{m}$ for small $n$. We obtain some general results on the $p$-primary part of the Farrell cohomology of $P \Gamma_{n(p-1) / 2}^{m}$ for some $m$, and the 2-primary part of the Farrell cohomology of the pure mapping class group $P \Gamma_{n}^{m}$ for $m=2 n-1,2 n, 2 n+1,2 n+2$, where $p$ is an odd prime and $n \geqslant 1$ is an integer. The explicit calculation is carried out for $\hat{H}^{i}\left(P \Gamma_{n(p-1) / 2}^{n+1}, \mathbb{Z}\right)_{(p)}, \hat{H}^{i}\left(P \Gamma_{n(p-1) / 2}^{n+2}, \mathbb{Z}\right)_{(p)}, \hat{H}^{i}\left(P \Gamma_{n}^{2 n+1}, \mathbb{Z}\right)_{(2)}$ and $\hat{H}^{i}\left(P \Gamma_{n}^{2 n+2}, \mathbb{Z}\right)_{(2)}$. The reason why we choose these groups is because the RiemannHurwitz formula implies the following lemma.

Lemma 5.2 Let $p$ be any odd prime, $m \geqslant 0$, and $n \geqslant 1$ be two integers.

(1) If $n-p+3 \leqslant m \leqslant n+2$, then every subgroup $\langle\alpha\rangle$ of order $p$ in $P \Gamma_{n(p-1) / 2}^{m}$ has $n+2$ fixed points including the punctures and the quotient surface $S_{n(p-1) / 2}^{m} /\langle\alpha\rangle$ is homeomorphic to $S_{0}^{m}$. 
(2) If $2 n-1 \leqslant m \leqslant 2 n+2$, then every subgroup $\langle\alpha\rangle$ of order 2 in $P \Gamma_{n}^{m}$ has $2 n+2$ fixed points including the punctures and the quotient surface $S_{n}^{m} /\langle\alpha\rangle$ is homeomorphic to $S_{0}^{m}$.

Remark 5.3 Lemma 5.2 is also a consequence of the main result of Chen-GloverJensen [7]: Every non-free orientable action of $\mathbb{Z} / p \mathbb{Z}$ on $S_{g}^{0}, p$ odd, is given by the connected sum at fixed points of the canonical actions of $\mathbb{Z} / p \mathbb{Z}$ on $S_{p}^{0}$ and $S_{(p-1) / 2}^{0}$. For $p=2$ the canonical action is for $\mathbb{Z} / 2 \mathbb{Z}$ on $S_{1}^{0}$ and $S_{2}^{0}$.

In view of Theorem 5.1, we start our computation by obtaining some structural information about the cohomology groups of normalizers of elements of prime order in $P \Gamma_{g}^{m}$ such that the quotient surface is homeomorphic to $S_{0}^{m}$.

Lemma 5.4 Let $\alpha \in P \Gamma_{g}^{m}$ be an element of prime order $p$ such that $S_{g}^{m} /\langle\alpha\rangle$ is homeomorphic to $S_{0}^{m}$, and $\Sigma_{\alpha}^{C}$ its centralizing permutation group. Let $N(\alpha)$ be the normalizer of $\alpha$ in $P \Gamma_{g}^{m}$. If $\Sigma_{\alpha}^{C}$ contains a subgroup of the form $K=H \times\{1\}^{l}$ for some $l \geqslant 3$, that is, $K$ fixes at least three punctures, and the index $\left[\Sigma_{\alpha}^{C}: K\right]$ is relatively prime to $p$, then $\hat{H}^{*}(N(\alpha), \mathbb{Z})_{(p)}$ is an elementary abelian group.

Proof By Theorem 4.6, the centralizer $C(\alpha)$ of $\alpha$ in $P \Gamma_{g}^{m}$ is isomorphic to the group $\operatorname{Aut}_{+}^{\Sigma_{\alpha}^{C}}\left(\pi_{1}\left(S_{0}^{n+m}\right)\right) / \operatorname{Inn}\left(\pi_{1}\left(S_{g}^{n+m}\right)\right)$, which will also be called $C(\alpha)$. Let $K(\alpha)=$ $\mathrm{Aut}_{+}^{K}\left(\pi_{1}\left(S_{0}^{n+m}\right)\right) / \operatorname{Inn}\left(\pi_{1}\left(S_{g}^{n+m}\right)\right)$. Then $[C(\alpha): K(\alpha)]=\left[\Sigma_{\alpha}^{C}: K\right]$ is prime to $p$. It follows that $[N(\alpha): K(\alpha)]=[N(\alpha): C(\alpha)][C(\alpha): K(\alpha)]$ is also prime to $p$. Since the group $K=H \times\{1\}^{l}$ and $l \geqslant 3$, by the algebraic description of the Artin braid group and the mapping class group and Theorem 3.3,

$$
\begin{aligned}
\operatorname{Aut}_{+}^{K}\left(\pi_{1}\left(S_{0}^{n+m}\right)\right) & =\left\langle B_{n+m-1}^{H \times\{1\}^{l-1}}, \operatorname{Inn}\left(\pi_{1}\left(S_{0}^{m+n}\right)\right)\right\rangle \\
& =\left\langle\Gamma_{0}^{n+m, K} \times \mathbb{Z}, \operatorname{Inn}\left(\pi_{1}\left(S_{0}^{m+n}\right)\right)\right\rangle,
\end{aligned}
$$

where the center $\mathbb{Z}=B_{n+m-1}^{H \times\{1\}^{l-1}} \cap \operatorname{Inn}\left(\pi_{1}\left(S_{0}^{m+n}\right)\right)$, and $\langle A, B\rangle$ denotes the subgroup generated by $A$ and $B$. Therefore,

$$
\begin{aligned}
K(\alpha) & =\operatorname{Aut}_{+}^{K}\left(\pi_{1}\left(S_{0}^{n+m}\right)\right) / \operatorname{Inn}\left(\pi_{1}\left(S_{g}^{m+n}\right)\right) \\
& =\left\langle B_{n+m-1}^{H \times\{1\}^{l-1}}, \operatorname{Inn}\left(\pi_{1}\left(S_{0}^{m+n}\right)\right)\right\rangle / \operatorname{Inn}\left(\pi_{1}\left(S_{g}^{m+n}\right)\right)
\end{aligned}
$$

is generated by the group $B_{n+m-1}^{H \times\{1\}^{l-1}} /\left[B_{n+m-1}^{H \times\{1\}^{l-1}} \cap \operatorname{Inn}\left(\pi_{1}\left(S_{g}^{m+n}\right)\right)\right]$ and the group $\operatorname{Inn}\left(\pi_{1}\left(S_{0}^{m+n}\right)\right) / \operatorname{Inn}\left(\pi_{1}\left(S_{g}^{m+n}\right)\right)=\langle\alpha\rangle$. Since

$$
B_{n+m-1}^{H \times\{1\}^{l-1}}=\Gamma_{0}^{n+m, K} \times\left(B_{n+m-1}^{H \times\{1\}^{l-1}} \cap \operatorname{Inn}\left(\pi_{1}\left(S_{0}^{m+n}\right)\right)\right),
$$


one has

$$
\begin{aligned}
& B_{n+m-1}^{H \times\{1\}^{l-1}} /\left[B_{n+m-1}^{H \times\{1\}^{l-1}} \cap \operatorname{Inn}\left(\pi_{1}\left(S_{g}^{m+n}\right)\right)\right]= \\
& \Gamma_{0}^{n+m, K} \times\left[\left(B_{n+m-1}^{H \times\{1\}^{l-1}} \cap \operatorname{Inn}\left(\pi_{1}\left(S_{0}^{m+n}\right)\right)\right) /\left(B_{n+m-1}^{H \times\{1\}^{l-1}} \cap \operatorname{Inn}\left(\pi_{1}\left(S_{g}^{m+n}\right)\right)\right)\right] .
\end{aligned}
$$

The group $\left(B_{n+m-1}^{H \times\{1\}^{l-1}} \cap \operatorname{Inn}\left(\pi_{1}\left(S_{0}^{m+n}\right)\right)\right) /\left(B_{n+m-1}^{H \times\{1\}^{l-1}} \cap \operatorname{Inn}\left(\pi_{1}\left(S_{g}^{m+n}\right)\right)\right)$ is a subgroup of $\left.\langle\alpha\rangle=\operatorname{Inn}\left(\pi_{1}\left(S_{0}^{m+n}\right)\right) / \operatorname{Inn}\left(\pi_{1}\left(S_{g}^{m+n}\right)\right)\right\rangle$ and therefore

$$
K(\alpha)=\Gamma_{0}^{n+m, K} \times\langle\alpha\rangle .
$$

By the Künneth formula, $H^{*}(K(\alpha), \mathbb{Z})$ is an elementary abelian group in sufficiently high dimension as $\Gamma_{0}^{n+m, K}$ has finite cohomological dimension. Since $K(\alpha)$ is $p$-periodic as it has $p$-rank $1, \hat{H}^{*}(K(\alpha), \mathbb{Z})$ is elementary abelian. According to [6, Proposition 9.5], the composition of restriction map $\operatorname{res}_{K(\alpha)}^{N(\alpha)}: \widehat{H}^{*}(N(\alpha), \mathbb{Z}) \longrightarrow$ $\hat{H}^{*}(K(\alpha), \mathbb{Z})$ with the transfer map $\operatorname{cor}_{N(\alpha)}^{K(\alpha)}: \hat{H}^{*}(K(\alpha), \mathbb{Z}) \stackrel{K(\alpha)}{\longrightarrow} \hat{H}^{*}(N(\alpha), \mathbb{Z})$ yields the scalar multiplication map multiplying with $[N(\alpha): K(\alpha)]$, that is,

$$
\operatorname{cor}_{N(\alpha)}^{K(\alpha)} \operatorname{res}_{K(\alpha)}^{N(\alpha)}(z)=[N(\alpha): K(\alpha)] z
$$

for all $z \in \hat{H}^{*}(N(\alpha), \mathbb{Z})$. Hence $\hat{H}^{*}(N(\alpha), \mathbb{Z})$ is annihilated by $p[N(\alpha): K(\alpha)]$ as $\operatorname{res}_{K(\alpha)}^{N(\alpha)}(p z)=p\left(\operatorname{res}_{K(\alpha)}^{N(\alpha)}(z)\right)=0$, and $\widehat{H}^{*}(N(\alpha), \mathbb{Z})_{(p)}$ is an elementary abelian group.

Corollary 5.5 Let $p$ be an odd prime and $\alpha \in P \Gamma_{n(p-1) / 2}^{m}$ an element of order $p$ with $n+2$ fixed points including punctures. Let $N(\alpha)$ be the normalizer of $\alpha$ in $P \Gamma_{n(p-1) / 2}^{m}$. If $m$ and $n$ satisfy

(i) $m \geqslant 3$; or

(ii) $1 \leqslant m \leqslant 2$ and $n \not \equiv 0(\bmod p)$; or

(iii) $m=0$ and $n \not \equiv 0$ or $-2(\bmod p)$,

then $\hat{H}^{*}(N(\alpha), \mathbb{Z})_{(p)}$ is an elementary abelian group.

Proof By the Riemann-Hurwitz formula, the quotient surface $S_{n(p-1) / 2}^{m} /\langle\alpha\rangle$ is homeomorphic to $S_{0}^{m}$. The case $m \geqslant 3$ follows rather obviously from Lemma 5.4. If $m=0$ and $n \neq 0$ or $-2(\bmod p)$, let $\Sigma_{\alpha}^{C}$ be the centralizing permutation group of $\alpha$. Then $\Sigma_{\alpha}^{C}=\Sigma_{l_{1}} \times \Sigma_{l_{2}} \times \cdots \times \Sigma_{l_{p-1}}$ with

$$
\begin{aligned}
& l_{1}+l_{2}+\cdots+l_{p-1}=n+2 \not \equiv 0(\bmod p) \\
& l_{1}+2 l_{2}+\cdots+(p-1) l_{p-1} \equiv 0(\bmod p) .
\end{aligned}
$$


If there are three $l_{i}$, say $l_{1}, l_{2}$ and $l_{3}$, prime to $p$, we can choose $H=\Sigma_{l_{1}-1} \times$ $\Sigma_{l_{2}-1} \times \Sigma_{l_{3}-1} \times \Sigma_{l_{4}} \cdots \times \Sigma_{l_{p-1}}$. If there are only two $l_{i}$, say $l_{1}$ and $l_{2}$, prime to $p$, then $\left(l_{1}-1\right)+\left(l_{2}-1\right) \equiv n \not \equiv 0(\bmod p)$ and there is at least one, say $l_{1}-1$, prime to $p$. Hence we can choose $H=\Sigma_{l_{1}-2} \times \Sigma_{l_{2}-1} \times \Sigma_{l_{3}} \times \cdots \times \Sigma_{l_{p-1}}$. By Lemma 5.4, $\hat{H}^{*}(N(\alpha), \mathbb{Z})_{(p)}$ is elementary abelian. The case $1 \leqslant m \leqslant 2$ and $n \not \equiv 0(\bmod p)$ can be similarly proved.

As we can see in most of the situations with quotient being the punctured 2-sphere, the $p$-primary part of Farrell cohomology has exponent $p$. By Lemma 5.2 and Corollary 5.5 , we have

Theorem 5.6 Let $m \geqslant 0$ and $n \geqslant 1$ be two integers.

(1) If $n-p+3 \leqslant m \leqslant n+2$ then $\hat{H}^{*}\left(P \Gamma_{n(p-1) / 2}^{m}, \mathbb{Z}\right)_{(p)}$ is an elementary abelian group.

(2) If $2 n-1 \leqslant m \leqslant 2 n+2$, then $\hat{H}^{*}\left(P \Gamma_{n}^{m}, \mathbb{Z}\right)_{(2)}$ is an elementary abelian group when $P \Gamma_{n}^{m} \neq P \Gamma_{1}^{1}$ or $P \Gamma_{1}^{2}$.

We are now ready to compute the groups $\hat{H}^{i}\left(P \Gamma_{n(p-1) / 2}^{n+1}, \mathbb{Z}\right)_{(p)}, \hat{H}^{i}\left(P \Gamma_{n(p-1) / 2}^{n+2}, \mathbb{Z}\right)_{(p)}$, $\hat{H}^{i}\left(P \Gamma_{n}^{2 n+1}, \mathbb{Z}\right)_{(2)}$ and $\hat{H}^{i}\left(P \Gamma_{n}^{2 n+2}, \mathbb{Z}\right)_{(2)}$. By Theorem 5.1, we need to find all conjugacy classes of subgroups of order $p$, which was done in $[11 ; 12]$. The idea is that there is an one to one correspondence between the conjugacy classes of subgroups $\langle\alpha\rangle$ of order $p$ in $P \Gamma_{n(p-1) / 2}^{n+1}$, as well as in $P \Gamma_{n(p-1) / 2}^{n+2}$, and the fixed point data vector up to scalers $\left\{k F_{\alpha} \mid k \in \mathbb{Z} / p \mathbb{Z}\right\}$. Hence the number of conjugacy classes of subgroups of order $p$ in $P \Gamma_{n(p-1) / 2}^{n+1}$ and in $P \Gamma_{n(p-1) / 2}^{n+2}$ is equal to the number of solutions to the equation $f\left(x_{1}\right)+f\left(x_{2}\right)+\cdots+f\left(x_{n+1}\right)=1$ in $\mathbb{Z} / p \mathbb{Z}$ and $f\left(x_{i}\right) \neq 0$ for all $i=1,2, \ldots, n+1$. If we denote the number of such solutions by $v(n+1)$, then we can get the following recursion formula based upon whether or not $f\left(x_{n+1}\right)=1$,

$$
(p-1) v(n-1)+(p-2) v(n)=v(n+1) .
$$

The initial values are obviously $v(1)=1$ and $v(2)=p-2$, By solving this recursion, one gets $v(n+1)=\left[(p-1)^{n+1}+(-1)^{n}\right] / p$. The groups $P \Gamma_{n}^{2 n+1}$ and $P \Gamma_{n}^{2 n+2}$ obviously have only one conjugacy class of subgroups of order 2 which fixes $2 n+2$ points. As a result we have the following lemma.

Lemma 5.7 The groups $P \Gamma_{n(p-1) / 2}^{n+1}$ and $P \Gamma_{n(p-1) / 2}^{n+2}$ have $\left[(p-1)^{n+1}+(-1)^{n}\right] / p$ conjugacy classes of subgroups of order $p$, and the groups $P \Gamma_{n}^{2 n+1}$ and $P \Gamma_{n}^{2 n+2}$ have 1 conjugacy class of subgroups of order 2 . 
If one follows the same line of calculation as in Lemma 5.4, then for any $\alpha \in P \Gamma_{n(p-1) / 2}^{n+1}$ or $\alpha \in P \Gamma_{n(p-1) / 2}^{n+2}$ of order $p$, one can see that

$$
N(\alpha)=C(\alpha)=K(\alpha)=P \Gamma_{0}^{n+2} \times \mathbb{Z} / p \mathbb{Z} .
$$

For any $\alpha \in P \Gamma_{n}^{2 n+1}$ or $\alpha \in P \Gamma_{n}^{2 n+2}$ of order 2 , one has

$$
N(\alpha)=C(\alpha)=K(\alpha)=P \Gamma_{0}^{2 n+2} \times \mathbb{Z} / 2 \mathbb{Z} .
$$

The cohomology groups of $P \Gamma_{0}^{m}$ have been computed by Cohen [8]. These are free abelian groups. The Poincaré series of the cohomology is given by

$$
P_{m}(t)=(1+2 t)(1+3 t) \cdots(1+(m-2) t) .
$$

Using the Künneth formula, one finds that

$$
\begin{aligned}
H^{i}(N(\alpha), \mathbb{Z})_{(p)} & =(\mathbb{Z} / p \mathbb{Z})^{\left(P_{n+2}(1)+(-1)^{i} P_{n+2}(-1)\right) / 2} \\
& =(\mathbb{Z} / p \mathbb{Z})^{\left(\frac{1}{2}(n+1) !+(-1)^{i+n-1}(n-1) !\right) / 2} \\
& =(\mathbb{Z} / p \mathbb{Z})^{(n-1) !\left[n^{2}+n-(-1)^{n+i} 2\right] / 4}
\end{aligned}
$$

for $i$ sufficiently large and $\alpha \in P \Gamma_{n(p-1) / 2}^{n+1}$ or $P \Gamma_{n(p-1) / 2}^{n+2}$, and

$$
\begin{aligned}
H^{i}(N(\alpha), \mathbb{Z})_{(2)} & =(\mathbb{Z} / 2 \mathbb{Z})^{\left(P_{2 n+2}(1)+(-1)^{i} P_{2 n+2}(-1)\right) / 2} \\
& =(\mathbb{Z} / 2 \mathbb{Z})^{\left(\frac{1}{2}(2 n+1) !+(-1)^{i+2 n-1}(2 n-1) !\right) / 2} \\
& =(\mathbb{Z} / 2 \mathbb{Z})^{(2 n-1) !\left[2 n^{2}+n-(-1)^{i}\right] / 2}
\end{aligned}
$$

for $i$ sufficiently large and $\alpha \in P \Gamma_{n}^{2 n+1}$ or $P \Gamma_{n}^{n+2}$. Since pure mapping class groups are of finite vcd, we know that their Farrell cohomology groups coincide with their ordinary cohomology groups above the vcd. Since pure mapping class groups are periodic, by Theorem 5.1 and Lemma 5.7, one has the following theorem.

Theorem 5.8 For any given odd prime $p$ and positive integer $n$, the $p$-primary part of the Farrell cohomology of $P \Gamma_{n(p-1) / 2}^{n+1}$ and $P \Gamma_{n(p-1) / 2}^{n+2}$ is given by

$$
\begin{aligned}
\hat{H}^{i}\left(P \Gamma_{n(p-1) / 2}^{n+1}, \mathbb{Z}\right)_{(p)} & \cong \hat{H}^{i}\left(P \Gamma_{n(p-1) / 2}^{n+2}, \mathbb{Z}\right)_{(p)} \\
& \cong(\mathbb{Z} / p \mathbb{Z})^{(n-1) !\left[(p-1)^{n+1}+(-1)^{n}\right]\left[n^{2}+n-(-1)^{n+i} 2\right] /(4 p)} .
\end{aligned}
$$

The 2-primary part of the Farrell cohomology of $P \Gamma_{n}^{2 n+1}$ and $P \Gamma_{n}^{2 n+2}$ is given by

$$
\hat{H}^{i}\left(P \Gamma_{n}^{2 n+1}, \mathbb{Z}\right)_{(2)} \cong \hat{H}^{i}\left(P \Gamma_{n}^{2 n+2}, \mathbb{Z}\right)_{(2)} \cong(\mathbb{Z} / 2 \mathbb{Z})^{(2 n-1) !\left[2 n^{2}+n-(-1)^{i}\right] / 2} .
$$


As illustrations of Theorem 5.8, we conclude this paper with the following remark.

Remark 5.9 (Lu [12; 13])

$$
\begin{aligned}
& \hat{H}^{0}\left(P \Gamma_{1}^{3}, \mathbb{Z}\right)_{(2)} \cong \hat{H}^{0}\left(P \Gamma_{1}^{4}, \mathbb{Z}\right)_{(2)} \cong \mathbb{Z} / 2 \mathbb{Z}, \\
& \hat{H}^{1}\left(P \Gamma_{1}^{3}, \mathbb{Z}\right)_{(2)} \cong \hat{H}^{1}\left(P \Gamma_{1}^{4}, \mathbb{Z}\right)_{(2)} \cong(\mathbb{Z} / 2 \mathbb{Z})^{2}, \\
& \hat{H}^{0}\left(P \Gamma_{(p-1) / 2}^{2}, \mathbb{Z}\right)_{(p)} \cong \hat{H}^{0}\left(P \Gamma_{(p-1) / 2}^{3}, \mathbb{Z}\right)_{(p)} \cong(\mathbb{Z} / p \mathbb{Z})^{p-2}, \\
& \hat{H}^{1}\left(P \Gamma_{(p-1) / 2}^{2}, \mathbb{Z}\right)_{(p)} \cong \hat{H}^{1}\left(P \Gamma_{(p-1) / 2}^{3}, \mathbb{Z}\right)_{(p)} \cong 0 \\
& \hat{H}^{0}\left(P \Gamma_{p-1}^{3}, \mathbb{Z}\right)_{(p)} \cong \hat{H}^{0}\left(P \Gamma_{p-1}^{4}, \mathbb{Z}\right)_{(p)} \cong(\mathbb{Z} / p \mathbb{Z})^{p^{2}-3 p+3}, \\
& \hat{H}^{1}\left(P \Gamma_{p-1}^{3}, \mathbb{Z}\right)_{(p)} \cong \hat{H}^{1}\left(P \Gamma_{p-1}^{4}, \mathbb{Z}\right)_{(p)} \cong(\mathbb{Z} / p \mathbb{Z})^{2\left(p^{2}-3 p+3\right)}, \\
& \hat{H}^{0}\left(P \Gamma_{3(p-1) / 2}^{4}, \mathbb{Z}\right)_{(p)} \cong \hat{H}^{0}\left(P \Gamma_{3(p-1) / 2}^{5}, \mathbb{Z}\right)_{(p)} \cong(\mathbb{Z} / p \mathbb{Z})^{7\left(p^{3}-4 p^{2}+6 p-4\right)}, \\
& \hat{H}^{1}\left(P \Gamma_{3(p-1) / 2}^{4}, \mathbb{Z}\right)_{(p)} \cong \hat{H}^{1}\left(P \Gamma_{3(p-1) / 2}^{5}, \mathbb{Z}\right)_{(p)} \cong(\mathbb{Z} / p \mathbb{Z})^{5\left(p^{3}-4 p^{2}+6 p-4\right)}
\end{aligned}
$$

\section{References}

[1] E Artin, Theorie der Zopfe, Hamburg Abh. 4 (1925) 47-72

[2] E Artin, Theory of braids, Ann. of Math. (2) 48 (1947) 101-126 MR0019087

[3] E Artin, Braids and permutations, Ann. of Math. (2) 48 (1947) 643-649 MR0020989

[4] J S Birman, Braids, links, and mapping class groups, Annals of Mathematics Studies 82, Princeton University Press, Princeton, N.J. (1974) MR0375281

[5] J S Birman, H M Hilden, On isotopies of homeomorphisms of Riemann surfaces, Ann. of Math. (2) 97 (1973) 424-439 MR0325959

[6] KS Brown, Cohomology of groups, Graduate Texts in Mathematics 87, Springer, New York (1982) MR672956

[7] Y Q Chen, H H Glover, CA Jensen, Prime order subgroups of mapping class groups (in preparation)

[8] F R Cohen, Artin's braid group and the homology of certain subgroups of the mapping class group, Mem. Amer. Math. Soc. 90 (1991) 6-28 MR1052554

[9] W Dicks, E Formanek, Automorphism subgroups of finite index in algebraic mapping class groups, J. Algebra 189 (1997) 58-89 MR1432365

[10] E Fadell, L Neuwirth, Configuration spaces, Math. Scand. 10 (1962) 111-118 MR0141126

[11] Q Lu, Cohomological properties of the punctured mapping class groups, $\mathrm{PhD}$ thesis, Ohio State University (1998) 
[12] Q Lu, Periodicity of the punctured mapping class group, J. Pure Appl. Algebra 155 (2001) 211-235 MR1801416

[13] Q Lu, Farrell cohomology of low genus pure mapping class groups with punctures, Algebr. Geom. Topol. 2 (2002) 537-562 MR1917066

[14] G Mislin, Mapping class groups, characteristic classes, and Bernoulli numbers, from: "The Hilton Symposium 1993 (Montreal, PQ)", CRM Proc. Lecture Notes 6, Amer. Math. Soc., Providence, RI (1994) 103-131 MR1290586

[15] Y Xia, The p-torsion of the Farrell-Tate cohomology of the mapping class group $\Gamma_{(p-1) / 2}$, from: “Topology '90 (Columbus, OH, 1990)", Ohio State Univ. Math. Res. Inst. Publ. 1, de Gruyter, Berlin (1992) 391-398 MR1184423

[16] H Zieschang, Finite groups of mapping classes of surfaces, Lecture Notes in Mathematics 875, Springer, Berlin (1981) MR643627

Department of Mathematics and Statistics, Wright State University,

Dayton, $\mathrm{OH} 45435-0001$

Department of Mathematics, Ohio State University,

Columbus, $\mathrm{OH} 43210-1174$

Department of Mathematics, University of New Orleans,

New Orleans, LA 70148

yuqing.chen@wright.edu, glover@math.ohio-state.edu, jensen@math.uno.edu

Received: 14 March $2007 \quad$ Revised: 16 September 2007 